\title{
Action for the infrared regime of gauge theories and the problem of color transformations
}

\author{
A. P. Balachandran ${ }^{1, *}$ and V.P. Nair ${ }^{2, \dagger}$ \\ ${ }^{1}$ Physics Department, Syracuse University Syracuse, New York 13244-1130, USA \\ ${ }^{2}$ Physics Department, City College of the CUNY New York, New York 10031, USA
}

(Received 21 April 2018; published 11 September 2018)

\begin{abstract}
It has been known for a while that there is spontaneous breaking of Lorentz symmetry in the nonzero charged sectors of quantum electrodynamics due to the infrared problem of soft photons. More recently, it has also been suggested that similar results hold for color transformations in a non-Abelian gauge theory. Here, we show that an action where a diffeomorphism has been carried out for the part describing hard gauge particles and matter fields can be used to analyze these issues. In addition to rederiving old results in this formalism, we also show that color transformations cannot be unitarily implemented on perturbative gluon states if gluon fields of arbitrarily low energy are allowed. Implications for confinement and mass gap are briefly commented upon.
\end{abstract}

DOI: 10.1103/PhysRevD.98.065007

\section{INTRODUCTION}

It has long been recognized that the existence of infrared divergences in a gauge theory leads to subtleties in the definition of charged states $[1,2]$. Charged particles are effectively accompanied by a cloud of soft photons. The analysis of scattering amplitudes shows that the charged states become non-Fock coherent states which may be defined by the action of a "dressing factor"on the Fock vacuum of soft photons $[1,3,4]$. The effect of this dressing on the S-matrix, specifically how it helps to factor out the infrared divergences, has become standard textbook material by now [2]. Nevertheless, this subject has seen a recent revival of interest related to the role of asymptotic symmetries and their consequences such as soft photon theorems in electrodynamics [5]. Another curious feature regarding the non-Fock coherent states which emerged from the extensive analysis carried out over many years is the spontaneous breaking of Lorentz symmetry in the nonzero charge sectors of the theory [6,7]. The dressing factor which leads to the coherent states is generated by the asymptotic soft radiation field associated to charged particles and is characterized by a timelike vector $p_{\mu}$. The overlap of such coherent states for different choices of this vector, i.e., for $p_{\mu}$ and $p_{\mu}^{\prime}$ (with $p_{\mu}^{\prime} \neq p_{\mu}$ ) is zero. In other

\footnotetext{
*balachandran38@gmail.com

†pnair@ccny.cuny.edu
}

Published by the American Physical Society under the terms of the Creative Commons Attribution 4.0 International license. Further distribution of this work must maintain attribution to the author(s) and the published article's title, journal citation, and DOI. Funded by SCOAP ${ }^{3}$. words, if we think of $p_{\mu}^{\prime}$ as a Lorentz transform of $p_{\mu}$, the corresponding transformation of the states cannot be unitarily implemented. While this spontaneous breaking must be taken account of in the theoretical set-up of quantum electrodynamics, its physical implications are less obvious. There is no such breaking in the sectors with zero net charge; since this sector is what is adequate for almost all practical situations (such as laboratory experiments on scattering), there is no easily obtainable observable consequence.

Given this situation, one possibility is to see if operators which are sensitive to this issue can be incorporated into the theory [8]. For example, in electrodynamics, one such operator is given by $U(\theta)=\exp (i Q(\theta))$ where

$$
Q(\theta)=\int d^{3} x\left(-\nabla \theta \cdot \vec{E}+\theta j_{0}\right)
$$

If $\theta$ is a smooth function on the spatial manifold $\mathbb{R}^{3}$ which vanishes at spatial infinity, then $Q(\theta)$ is just the Gauss law and hence it will vanish on physical states. For a function $\theta$ which becomes a constant (say $\theta_{\infty}$ ) at spatial infinity, $Q(\theta)$ becomes $\theta_{\infty}$ times the charge operator, while for those functions $\theta$ which tend to nontrivial functions on the twosphere at spatial infinity, $Q(\theta)$ furnish a set of operators sensitive to the asymptotic behavior of the fields. One can attempt modifying the theory in a way which depends on these; e.g., a modified mass term $m \rightarrow m\left(U(\theta)+U^{\dagger}(\theta)\right)$ $[8,9]$. Interference effects may be another way to detect consequences of the infrared dressing factor [10].

Another set of questions arises from the familiar consequences of spontaneous symmetry breaking. Is there a Goldstone mode one can identify? Also, recall that while 
the Goldstone theorem and the Higgs mechanism can be explained in terms of correlators of operators, there is a simple action-based description which captures the essence of the phenomena. One can ask if there is a similar description in the present case. Finally, in a non-Abelian gauge theory, where the charge algebra is non-Abelian, the dressing factor is applied to a specific charged state of a representation of this algebra. The characterization of the state by the charge is similar to the characterization of the state by the vector $p_{\mu}$ as regards Lorentz transformations. Thus, one can ask if, in a similar way to what happens with Lorentz transformations, the unitary realization of charge (or color) rotations is vitiated by the infrared properties of the dressing factor. These are the questions of interest in this paper. We note that the possibility of breaking of color rotations has been suggested and arguments in its favor given previously [9].

In a charged sector of a gauge theory like electrodynamics, we have a collection of charged particles described by matter fields. We can construct an action where we separate out the coupling of the soft photons to these charged particles. This is explained in the next section. The action is further justified by showing that it leads to the dressing factor and the vanishing of the overlap of states with different Lorentz transforms of the defining timelike vector $p_{\mu}$. This is carried out in Sec. III. In Sec. IV, we give the generalization to non-Abelian gauge fields. The color rotations are constructed, and, in Sec. V, in an approximation spelled out in detail there, we show the vanishing of the overlap of color-rotated states. Some of the details which are technical and not essential to the flow of logic are relegated to three Appendices.

The problem of color rotations is clearly of import to the question of color confinement. We end the paper with a short discussion of this matter. Previous work by Balachandran and collaborators, including the discussion of the breaking of Lorentz and color transformations, are in $[8,9]$. The present paper has occasional overlap with those papers.

\section{AN ACTION FOR INFRARED DYNAMICS}

One approach to the infrared dynamics is via the algebra of local observables and careful analysis of the asymptotic behavior, as carried out in many papers, see e.g., [6-8] and references therein. Perhaps a more physical point of view is obtained by noting that, basically, in a charged sector of the theory we have a collection of charged particles (described by matter fields) with a net charge not equal to zero. We may think of this cluster of particles as a single composite particle or as a droplet of charged matter fields. In terms of coupling to the photon, it is well known that the low energy photons (with $\omega \sim|\vec{k}| \rightarrow 0$ ) only couple to the overall charge, the monopole moment of the charge distribution, while photons of higher energy couple to the higher moments of the charge distribution via $F_{\mu \nu}$ and its derivatives. The soft photons decouple from the higher moments as $\vec{k} \rightarrow 0$ since they involve $F_{\mu \nu}$ and its derivatives. Thus, it should be possible to separate out the overall dynamics of the system of charged particles and hard photons from the infrared photons. We will now consider writing the action for the theory in a form which displays this separation of the infrared dynamics, starting with electrodynamics.

For clarity, we will use $A_{\mu}$ as the gauge potential for the hard photons and $a_{\mu}$ for the soft photons, with the corresponding field strengths $F_{\mu \nu}=\partial_{\mu} A_{\nu}-\partial_{\nu} A_{\mu}, f_{\mu \nu}=\partial_{\mu} a_{\nu}-$ $\partial_{\nu} a_{\mu}$. The action is then given by

$$
\begin{aligned}
S & =S_{1}+S_{2}+S_{3} \\
S_{1}(\Lambda, \lambda) & =-\frac{1}{4} \int d^{4} x \sqrt{-g} g^{a c} g^{b d} F_{a b} F_{c d}+S_{\text {matter }}(g) \\
S_{2}(\lambda, \mu) & =-\frac{1}{4} \int d^{4} x \sqrt{-\eta} \eta^{\mu \alpha} \eta^{\nu \beta} f_{\mu \nu} f_{\alpha \beta} \\
S_{3} & =-q \int a_{\mu}(Z) d Z^{\mu}
\end{aligned}
$$

This action needs a number of explanatory remarks. In (2), we separate the fields in terms of the range of momenta involved. $S_{1}$ consists of modes of photons with wave vectors $\vec{k}$, with $\lambda<|\vec{k}| \leq \Lambda$. Thus, $\Lambda$ is an upper cutoff for the whole theory and $\lambda$ designates the separation between the hard photons and the soft photons. This action has the standard form, except that the metric is given by

$d s^{2}=d Z_{0}^{2}-\left(d x^{i}+d Z^{i}\right)^{2}=\dot{Z}_{0}^{2} d t^{2}-\left(d x^{i}+\dot{Z}^{i} d t\right)^{2}$.

Here $Z^{\mu}$ can be viewed as the center of mass coordinate for the system of hard photons and matter fields which form the overall composite particle or droplet. If we compare this with the usual splitting of the metric into the 3-metric $\sigma_{i j}$ and lapse $(\alpha)$ and shift $\left(\beta^{i}\right)$ functions, which is given by

$$
d s^{2}=\alpha^{2} d t^{2}-\sigma_{i j}\left(d x^{i}+\beta^{i} d t\right)\left(d x^{j}+\beta^{j} d t\right),
$$

we see that (3) is equivalent to $\alpha=\dot{Z}_{0}, \dot{Z}_{i}=\beta_{i}$ and $\sigma_{i j}=\delta_{i j}$. Thus, we may think of $S_{1}$ as obtained from the standard form of the action for electrodynamics (but with an upper and lower cutoff on the range of momenta) by a diffeomorphism $\left(x^{0}, x^{i}\right) \rightarrow\left(Z^{0}, x^{i}+Z^{i}\right)$. In other words, $S_{1}$ has the form of a parametrized field theory as in [11]. If we carry out such a diffeomorphism, $S_{1}$ being the standard QED action, we will obtain the usual results of QED, as it should, only qualified by the presence of the cutoffs. (The limit of vanishingly small $\lambda$ may be taken at the end for various infrared finite quantities calculated using this part of the action. The precise matching between the hard and soft modes will not be important since we take this limit at the end.) It is also worth pointing out that, if the entire action were just $S_{1}$, there would be no nontrivial equations of motion for $Z^{\mu}$ since it can be eliminated by a diffeomorphism. But because of the additional terms $S_{2}+S_{3}$, the results will depend on $Z^{\mu}$ or rather $\dot{Z}^{\mu}$ as we shall see below. 
The second part of the action, $S_{2}$, describes the soft photons; it has an upper cutoff of $\lambda$ and a lower cutoff of $\mu$; eventually we will take $\mu \rightarrow 0$. (If we take $\lambda \rightarrow 0$ for infrared finite quantities, the correct order is to take $\mu \rightarrow 0$ first and then $\lambda \rightarrow 0$.) We consider flat ambient spacetime, with $\eta_{\mu \nu}$ as the Minkowski metric. The third term of the action, $S_{3}$, gives the coupling of the "droplet"of hard photons and charged fields, treated as single composite particle, to the soft photons.

We assume that there is some gauge-invariant way of introducing the cutoffs; the details of this are not relevant for our discussion. Further, we will take matter fields to be massive so that there are no additional infrared problems due to a vanishing mass for the charged particles. $S_{1}$ is the term involving the metric $g_{\mu \nu}$, which has $Z$-dependence. $S_{3}$, thus, couples the modes in $S_{1}$ to the soft photons. However, if we consider $S_{1}$ in terms of $\left(Z^{0}, x^{i}+Z^{i}\right)$, it is just the standard QED (with a lower cutoff) coupled to charged particles, so up to a diffeomorphism, the theory of hard photons and charged particles is standard QED without infrared divergences. (We also note that lapse and shift functions are also central to the analysis of the BMS symmetry and soft modes for gravitons [12-14].)

For facility of calculations, we may note at this point that the metric tensor appearing in (3) and its inverse are given by

$$
\begin{aligned}
g_{a b} & =\left[\begin{array}{cc}
\dot{Z}_{0}^{2}-\dot{Z}_{i}^{2} & -\dot{Z}_{i} \\
-\dot{Z}_{i} & -\delta_{i j}
\end{array}\right], \\
g^{a b} & =\frac{1}{\dot{Z}_{0}^{2}}\left[\begin{array}{cc}
1 & -\dot{Z}_{i} \\
-\dot{Z}_{i} & -\delta_{i j} \dot{Z}_{0}^{2}+\dot{Z}_{i} \dot{Z}_{j}
\end{array}\right],
\end{aligned}
$$

with $\operatorname{det} g=-\dot{Z}_{0}^{2}$.

We have given the motivating arguments for the form of the action in (2). But ultimately, (2) is to be taken as the starting postulate of our analysis. The real reason for it is that it reproduces known results for the dressing factor for charged states in terms of the soft photons and the spontaneous breakdown of Lorentz symmetry as in $[6,7]$. To demonstrate this and work out the consequences of (2), we will analyze the dynamics in a combination of the pathintegral and Hamiltonian approaches. But, as a first step, it is useful to consider the classical equations of motion for the soft photons. The variation of $X^{\mu}=\left(Z^{0}, x^{i}+Z^{i}\right)$ or $Z^{\mu}$ gets contributions from $S_{1}$ (via the induced metric $g_{a b}$ ) and $S_{3}$, while variations in $a_{\mu}$ have contributions from $S_{2}$, $S_{3}$. The variational equations are given by

$$
\begin{aligned}
\frac{1}{\sqrt{-g}} \partial_{a}\left[\sqrt{-g} T^{a b} \eta_{\alpha \nu} \partial_{b} X^{\nu}\right] & =f_{\alpha \mu} J^{\mu} \\
\partial_{\mu} f^{\mu \nu} & =J^{\nu} \\
J^{\nu}(y) & =q \int d \tau \frac{d Z^{\mu}}{d \tau} \delta^{(4)}(y-Z(\tau)) .
\end{aligned}
$$

Here $T^{a b}$ is the energy-momentum tensor for the matter fields and the hard modes of the electromagnetic field. Here we have a coupling of $S_{1}$ to $Z^{\mu}$ via $S_{3}$, so we do not expect conservation of $T^{a b}$. Since $X^{\nu}$ is a diffeomorphism of $x^{\mu}$, for this term, we can go back to the $X$-coordinates and write

$$
\partial_{b} x^{\nu} \frac{1}{\sqrt{-g}} \frac{\partial}{\partial \xi^{a}}\left(\sqrt{-g} T^{a b}\right)=\frac{\partial}{\partial x^{\mu}} T^{\mu \nu} .
$$

Further, the second equation in (6), which is the equation of motion for $a_{\mu}$, gives

$$
\partial_{\mu} t^{\mu \nu}=-f^{\nu \mu} J_{\mu}
$$

where $t^{\mu \nu}$ is the energy-momentum tensor for the electromagnetic field. It is defined as $t^{\mu \nu}=f^{\mu \alpha} f_{\alpha}^{\nu}+\frac{1}{4} \eta^{\mu \nu} f^{2}$. Conservation of energy-momentum for the whole theory reduces to $\partial_{\mu}\left(T^{\mu \nu}+t^{\mu \nu}\right)=0$.

Finally, we will consider $Z^{\mu}$ such that $\left(d Z^{\mu} / d \tau\right)=p^{\mu}$, where $p^{\mu}$ is a constant timelike vector. This is what we expect from the interpretation of the system of charged particles and hard photons as a composite particle. Consistency of this choice will require that, if we take the limit of $\lambda$ as well as $\mu$ becoming zero, the energy loss due to the radiation of soft photons should go to zero and we should get $\partial_{\mu} T^{\mu \nu}=0$, which ensures that the droplet has a conserved total momentum. This is indeed the case as shown in the Appendix.

The key result in this section is the action (2). We will now use it to construct the dressing factor for charged states.

\section{THE DRESSING FACTOR AND BREAKING OF LORENTZ SYMMETRY}

We start with the action (2) focusing on the dynamics of the soft photons described by $S_{2}+S_{3}$ since this is sufficient for deriving the dressing factor. Consider quantizing this theory using functional integrals. For simplicity, we use the gauge $a_{0}=0$ and $\nabla \cdot a=0$. States can then be represented by wave functionals of $a_{i}$ (which is transverse). The transition matrix element between states $|\alpha\rangle$ and $|\beta\rangle$ is given by

$$
\left\langle\alpha\left|e^{-i H t}\right| \beta\right\rangle=\int[d a] \Psi_{\alpha}^{*}\left(a^{\prime}\right) e^{i S[a])} \Psi_{\beta}\left(a^{\prime \prime}\right),
$$

where $[d a]$ is the appropriate gauge-invariant measure and the configurations which are integrated over have $a(t)=a^{\prime \prime}$ at $t=0$ and $a(t)=a^{\prime}$ at the final value of time $t . \Psi_{\alpha}\left(a^{\prime}\right)$ and $\Psi_{\beta}\left(a^{\prime \prime}\right)$ are the wave functionals for the final and initial states, respectively, corresponding to the times $t$ and 0 . The action $S[a]=S_{2}+S_{3}$ with the addition of the needed gauge fixing. 
Consider now the action where we shift the variable as $a \rightarrow \hat{a}+a^{(c)}$, where $a^{(c)}$ is a background $c$-number function. This will be chosen to obey the equations of motion,

$$
\partial_{\mu}\left(f^{(c)}\right)^{\mu \nu}=J^{\nu}
$$

The action $S_{2}(\lambda, \mu)+S_{3}(\lambda, \mu)$ simplifies as

$$
\begin{aligned}
S\left[\hat{a}+a^{(c)}\right]= & -\frac{1}{4} \int \hat{f}^{2}-\frac{1}{2} \int\left(f^{(c)}\right)^{\mu \nu} \hat{f}_{\mu \nu}-\frac{1}{4} \int\left(f^{(c)}\right)^{2} \\
& -\int \hat{a}_{\mu} J^{\mu}-\int\left(a^{(c)}\right)^{\mu} J_{\mu} \\
= & -\frac{1}{4} \int \hat{f}^{2}-\oint \hat{a}_{\nu}\left(f^{(c)}\right)^{\mu \nu} d S_{\mu} \\
& +\int \hat{a}_{\nu}\left(\partial_{\mu}\left(f^{(c)}\right)^{\mu \nu}-J^{\nu}\right)+\cdots \\
= & \left.-\frac{1}{4} \int \hat{f}^{2}+\int d^{3} x e_{i}^{(c)} \hat{a}_{i}\right]_{0}^{t}+\cdots,
\end{aligned}
$$

where $e_{i}^{(c)}$ is the electric field corresponding to $a_{i}^{(c)}$ and the ellipsis denotes terms which are independent of $\hat{a}$ and hence constants which factor out of the functional integral. There are gauge fixing terms for the $\hat{a}$-fields which are understood as being added to the expression shown. Using the result (11), we can simplify (9) as

$$
\begin{aligned}
\left\langle\alpha\left|e^{-i H t}\right| \beta\right\rangle= & \int[d \hat{a}]\left[\Psi_{\alpha}^{*}\left(\left(\hat{a}+a^{(c)}\right)^{\prime}, t\right) e^{i \int e^{(c)} \hat{a}(t)}\right] \\
& \times e^{\left.i S_{0}[\hat{a}]\right)}\left[e^{-i \int e^{(c)} \cdot \hat{a}(0)} \Psi_{\beta}\left(\left(\hat{a}+a^{(c)}\right)^{\prime \prime}, 0\right)\right],
\end{aligned}
$$

where $S_{0}[\hat{a}]=-\frac{1}{4} \int \hat{f}^{2}$. This equation shows that, as far as the dynamics of the soft photons is concerned, we have a free Maxwell theory (governed by $S_{0}[\hat{a}]$ ) and hence its contribution to correlators of hard photons and matter will factor out. However, the incoming and outgoing states must be given by

$$
\begin{aligned}
\langle\hat{a} \mid \tilde{\beta}\rangle & =e^{-i \int e^{(c)} \cdot \hat{a}} \Psi_{\beta}\left(\hat{a}+a^{(c)}\right) \\
& =e^{-i \int e^{(c)} \hat{a}} e^{i \int a^{(c)} \cdot \hat{e}} \Psi_{\beta}(\hat{a}) \\
& =V\left(a^{(c)}, e^{(c)}\right) \Psi_{\beta}(\hat{a})=\left\langle a\left|V\left(a^{(c)}, e^{(c)}\right)\right| \beta\right\rangle,
\end{aligned}
$$

where

$$
V\left(a^{(c)}, e^{(c)}\right)=\exp \left[-i \int\left(e^{(c)} \cdot \hat{a}-a^{(c)} \cdot \hat{e}\right)\right] .
$$

Here we have translated the wave functionals to the operator language using $\Psi_{\beta}(\hat{a})=\langle\hat{a} \mid \beta\rangle$, using the notation $|\tilde{\beta}\rangle$ for the redefined state. This allows us to obtain the operator expression for the dressing factor $V\left(a^{(c)}, e^{(c)}\right)$. In (13), (14), $\hat{e}_{i}$ is the operator conjugate to $\hat{a}_{i}$, so that $\exp \left[i \int a^{(c)} \cdot \hat{e}\right]$ can be used to shift the field $\hat{a}+a^{(c)}$ to $\hat{a}$ in $\Psi$. There can be normal ordering corrections in going from the second to last line in (13); this has been absorbed into the normalization of the wave functionals. $V\left(a^{(c)}, e^{(c)}\right)$ gives the dressing factor for the states due to the soft photon modes. If the initial and final states in (9) are the vacuum states, we can take them to be the Fock vacua for the soft photons. But the action of the formally unitary operator $V\left(a^{(c)}, e^{(c)}\right)$ converts them to coherent states defined by the classical functions $a_{i}^{(c)}$ and $e_{i}^{(c)}$. The full computation of the amplitude now reduces to the computation with the hard photons and the matter fields with the dressing factor $V$ included for the incoming and outgoing states. Since the action for the hard photons has a lower cutoff of $\lambda$, we see that there will be no infrared divergences in the correlations functions for the hard photons and matter fields as $\mu \rightarrow 0$.

We now turn to the issues with the unitary implementation of Lorentz transformations. This result can be seen by considering the overlap of the coherent states defined above. The operators $\hat{a}_{i}$ and $\hat{e}_{i}$ have the mode expansion

$$
\begin{aligned}
& \hat{a}_{i}=\sum_{k} \frac{1}{\sqrt{2 \omega_{k} \mathcal{V}}}\left(c_{i}(k) e^{-i k x}+c_{i}^{\dagger}(k) e^{i k x}\right) \\
& \hat{e}_{i}=\sum_{k} \frac{\left(-i \omega_{k}\right)}{\sqrt{2 \omega_{k} \mathcal{V}}}\left(c_{i}(k) e^{-i k x}-c_{i}^{\dagger}(k) e^{i k x}\right),
\end{aligned}
$$

where $\omega_{k}=\sqrt{\vec{k} \cdot \vec{k}}$ and $c_{i}(k), c_{i}^{\dagger}(k)$ are the usual annihilation and creation operators for the photons. (Here we have used a discrete sum over $\vec{k}$ rather than an integral, because this is convenient for calculations. We take fields in a cubical volume $\mathcal{V}=L^{3}$ with periodic boundary conditions for the fields, so that $\vec{k}=2 \pi\left(n_{1}, n_{2}, n_{3}\right) / L$, $n_{i} \in \mathbb{Z}$. Eventually, we take the limit $L \rightarrow \infty$ in the usual way.) The summation (or integration as $\mathcal{V} \rightarrow \infty$ ) is over the range $\mu \leq|\vec{k}| \leq \lambda$. The operator $V\left(a^{(c)}, e^{(c)}\right)$ in (14) can be explicitly written out in terms of these operators as $V=e^{\Phi}$ with

$$
\begin{aligned}
\Phi & =-i \int\left(e^{(c)} \cdot \hat{a}-a^{(c)} \cdot \hat{e}\right) \\
& =q \sum_{k} \frac{1}{\sqrt{2 \omega_{k} \mathcal{V}}}\left[\Omega_{i}^{+}(-k, p) c_{i}(k)-\Omega_{i}^{-}(k, p) c_{i}^{\dagger}(k)\right] \\
\Omega_{i}^{ \pm}(k, p) & =\left(p_{i}-k_{i} \frac{\vec{k} \cdot \vec{p}}{\vec{k}^{2}}\right) \frac{1}{p k \pm i \epsilon} .
\end{aligned}
$$

Here, $p k=p_{0} k_{0}-\vec{p} \cdot \vec{k}$. The dressed states are characterized by the vector $\vec{p}$ as it appears in $\Omega^{ \pm}$; so we will designate the dressed state for the Fock vacuum of soft photons as $|\vec{p}\rangle$. Carrying out the normal ordering for $V$, we then get 


$$
\begin{aligned}
|\vec{p}\rangle & =\exp \left[-\frac{q^{2}}{2}\left(\Omega^{+}, \Omega^{-}\right)-q \sum_{k} \frac{1}{\sqrt{2 \omega_{k} \mathcal{V}}} \Omega^{-} \cdot c^{\dagger}\right]|0\rangle \\
\left(\Omega^{+}, \Omega^{-}\right) & =\sum_{k} \frac{1}{2 \omega_{k} \mathcal{V}} \Omega_{i}^{+}(k, p) \Omega_{i}^{-}(k, p) \\
& =\int \frac{d^{3} k}{(2 \pi)^{3}} \frac{1}{2 \omega_{k}} \Omega_{i}^{+}(k, p) \Omega_{i}^{-}(k, p) .
\end{aligned}
$$

In the last expression, we have taken the $\mathcal{V} \rightarrow \infty$ limit. And, once again, the range of integration is restricted to $\mu \leq|\vec{k}| \leq \lambda$. It is easily checked that this state is normalized, $\langle\vec{p} \mid \vec{p}\rangle=1$.

The overlap of states for $\vec{p}$ and $\vec{p}^{\prime}$ is given by

$$
\begin{aligned}
\left\langle\vec{p}^{\prime} \mid \vec{p}\right\rangle= & \exp \left[-\frac{q^{2}}{2}\left(\Omega_{p}^{+}, \Omega_{p}^{-}\right)-\frac{q^{2}}{2}\left(\Omega_{p^{\prime}}^{+}, \Omega_{p^{\prime}}^{-}\right)\right. \\
& \left.+q^{2}\left(\Omega_{p^{\prime}}^{+}, \Omega_{p}^{-}\right)\right] \\
\left|\left\langle\vec{p}^{\prime} \mid \vec{p}\right\rangle\right|^{2}= & \exp \left[-q^{2}\left(\Omega_{p}^{+}-\Omega_{p^{\prime}}^{+}, \Omega_{p}^{-}-\Omega_{p^{\prime}}^{-}\right)\right] .
\end{aligned}
$$

The integration over the magnitude of $\vec{k}$ can be carried out to write

$$
\begin{aligned}
\left(\Omega_{p^{\prime}}^{+}, \Omega_{p}^{-}\right) & =\frac{1}{16 \pi^{3}} \log (\lambda / \mu)\left(\vec{v}^{\prime}, \vec{v}\right) \\
\left(\vec{v}^{\prime}, \vec{v}\right) & =\int d \Omega \frac{\left(v_{i}^{\prime}-\hat{k}_{i} \vec{v}^{\prime} \cdot \hat{k}\right)}{1-\vec{v}^{\prime} \cdot \hat{k}+i \epsilon} \frac{\left(v_{i}-\hat{k_{i}} \vec{v} \cdot \hat{k}\right)}{1-\vec{v} \cdot \hat{k}+i \epsilon},
\end{aligned}
$$

where $\vec{v}=\vec{p} / p_{0}, \vec{v}^{\prime}=\vec{p}^{\prime} / p_{0}^{\prime}$, and the remaining integration is over the angular degrees of freedom in $\hat{k}=\vec{k} /|\vec{k}|$. The bracket $\left(\vec{v}^{\prime}, \vec{v}\right)$ may be viewed as an inner product for the velocities $\vec{v}$ and $\vec{v}^{\prime}$. It is positive semidefinite. Thus, from (18), we see that

$\left|\left\langle\vec{p}^{\prime} \mid \vec{p}\right\rangle\right|^{2}=\exp \left[-\frac{q^{2}}{16 \pi^{3}} \log (\lambda / \mu)\left(\vec{v}-\vec{v}^{\prime}, \vec{v}-\vec{v}^{\prime}\right)\right]$

This vanishes as $\mu \rightarrow 0$ at fixed $\lambda$, for any choice of $\vec{v} \neq \vec{v}^{\prime}$. Regarding $p_{\mu}^{\prime}$ as obtained by a Lorentz transformation of $p_{\mu}$, this overlap is equivalent to calculating the matrix element of the operator corresponding to the Lorentz transformation for the dressed soft photon states; i.e., if $p_{\mu}^{\prime}=\Lambda_{\mu}^{\nu} p_{\nu}$, then $\left\langle\vec{p}^{\prime} \mid \vec{p}\right\rangle=\left\langle\vec{p}\left|U_{\Lambda}\right| \vec{p}\right\rangle$. The vanishing of this matrix element is, thus, equivalent to the statement that the Lorentz transformations cannot be unitarily implemented on the dressed states. It is also straightforward to show that the matrix elements of all local operators, such as integrals of creation-annihilation operators smeared over non-zero momenta, will also vanish as $\mu \rightarrow 0$. Together, these statements constitute the spontaneous breaking of Lorentz symmetry discussed in [6-8].
The physical meaning of this breaking is also clear from our derivation. It is simply that the total momentum of the droplet of charged particles and hard photons is a superselected parameter, and cannot be changed by any operator action due to the soft modes. As we remarked earlier, the correlation functions of local operators do not manifest this breaking of Lorentz symmetry in the zero charge sector where $q^{2}=0$. This sector is what is relevant for the calculation of the S-matrix elements of QED in flat space for practical applications, so standard experiments will not detect this. It can only be detected if there are other forces such as a gravitational field which can affect the overall motion of the droplet, or in carefully designed experiments for detecting phase information [10].

To briefly recapitulate, in this section, starting from the action (2), we have obtained the dressing factor and the coherent states for soft photons; these are given in (13), (14). Further, by considering the overlap of such coherent states, specifically as in (20), we have rederived the known result on spontaneous breaking of Lorentz symmetry in electrodynamics.

\section{ACTION AND DRESSING FACTOR FOR NON-ABELIAN GAUGE THEORY}

Generalizing from the electromagnetic case, the action for the non-Abelian gauge theory, with the coupling to the lapse and shift functions, can be written down in a straightforward way as

$$
\begin{aligned}
S & =S_{1}+S_{2}+S_{3}+S_{4} \\
S_{1} & =-\frac{1}{4} \int d^{4} x \sqrt{-g} g^{a c} g^{b d} F_{a b}^{A} F_{c d}^{A} \\
S_{2} & =-\frac{1}{4} \int d^{4} x \sqrt{-\eta} \eta^{\mu \alpha} \eta^{\nu \beta} f_{\mu \nu}^{A} f_{\alpha \beta}^{A} \\
S_{3} & =-\int d \tau Q_{A} a_{\mu}^{A} \frac{d Z^{\mu}}{d \tau} \\
S_{4} & =i \int d \tau \sum_{k} w_{k} \operatorname{Tr}\left(t_{k} u^{-1} \dot{u}\right)
\end{aligned}
$$

The superscripts $A$ denote the color components, corresponding to a basis of the Lie algebra of the color group $G$ in which the gauge transformations take values. $u$ is an element of $G$ and $Q_{A}=2 \sum w_{k} \operatorname{Tr}\left(t_{k} u^{-1} t_{A} u\right), t_{A}$ being the generators of the group in the chosen basis for the Lie algebra. Our choice of normalization for $t_{A}$ is $\operatorname{Tr}\left(t_{A} t_{B}\right)=$ $\frac{1}{2} \delta_{A B} . t_{k}$ are the diagonal generators in the same basis. The term $S_{4}$ leads to a representation of the color group with highest weight vector $\left(w_{1}, w_{2}, \ldots, w_{r}\right)$ upon quantization, $r$ being the rank of the algebra [15-17].

As in the Abelian case, the wave vectors for the fields are restricted to be in the range $\lambda \leq|\vec{k}|<\Lambda$ for $S_{1}$, and $\mu \leq$ $|\vec{k}|<\lambda$ for $S_{2}, S_{3}$. In the Abelian case, we were able to take the limit $\mu \rightarrow 0$, which led to the orthogonality of the 
dressed states for different values of the timelike vector $p_{\mu}$ which characterizes them. In the non-Abelian case, even in perturbation theory, we have a problem since there are many self-interactions for the gauge field. If we want to use perturbation theory, generally because of asymptotic freedom, $\mu$ must be larger than some scale factor like $\Lambda_{\mathrm{QCD}}$ which defines the theory. However, from (20), we see that we need $\mu \rightarrow 0$ to obtain the orthogonality of states. Therefore, we do not have an obvious kinematic regime where we can use perturbation theory and make statements regarding the orthogonality of states due to the dressing factor, even to the lowest order in the perturbative expansion. One option is to consider a theory with sufficient number of matter fields so that we do not have asymptotic freedom. One can then pose the question of whether color and Lorentz transformations can be implemented within perturbation theory in such a model. We will show that color transformations cannot be unitarily implemented in such a theory, in the sectors with nonzero color charges, at least for the case when $p_{i} \neq 0$. Lorentz transformations cannot be unitarily implemented either, but that is essentially the same as in electrodynamics, so we focus on the color rotations.

Returning to the action, our focus is on the low energy modes corresponding to the action $S_{2}+S_{3}+S_{4}$. As before, we will consider the shift

$$
a_{\mu}^{A}=\hat{a}_{\mu}^{A}+\left(a^{(c)}\right)_{\mu}^{A}
$$

In the Abelian case, $a_{\mu}^{(c)}$ was a $c$-number field determined in terms of the source $\dot{Z}^{\mu}$. In the present case, because the source is $Q_{A} \dot{Z}^{\mu},\left(a^{(c)}\right)_{\mu}^{A}$ will involve $Q_{A}$ which is the color charge operator when we quantize $u \in G$. So $\left(a^{(c)}\right)_{\mu}^{A}$ will no longer be a $c$-number function.

We may think of the whole quantization procedure (for both the fields and for $g$ ) in the Hamiltonian formalism with states described by wave functionals of $a_{i}^{A}$ and $g$,

$$
\Psi_{\alpha, A}(a, g)=\langle a, g \mid \alpha, r\rangle
$$

corresponding to a state $|\alpha\rangle$ labeling momenta, polarization, etc. and color state $|r\rangle$. We used a functional integral approach to identify the dressing factor in the Abelian case. However, here, because of the ghosts which may not factor out, there is no particular advantage to the functional integral. We will use a Hamiltonian approach with $a_{0}^{A}=0$. (The previous results will also be easily recovered within this framework.) The Hamiltonian for $S_{2}+S_{3}+S_{4}$ is given by

$$
\begin{aligned}
H & =\frac{1}{2} \int\left(e^{2}+b^{2}\right)+a_{i}^{A} Q_{A} \dot{Z}^{i} \\
e_{i}^{A} & =\dot{a}_{i}^{A}, \quad b_{i}^{A}=\frac{1}{2} \epsilon_{i j k}\left(\partial_{j} a_{k}^{A}-\partial_{k} a_{j}^{A}+f^{A B C} a_{j}^{B} a_{k}^{C}\right)
\end{aligned}
$$

The time-evolution operator is obviously given by $U(t, 0)=T \exp \left(-i \int_{0}^{t} d t H\right)$. We now write this as $U(t, 0)=$ $V(t)^{-1} \tilde{U}(t, 0) V(0)$ where

$$
\begin{aligned}
\tilde{U}(t, 0) & =T \exp \left(-i \int_{0}^{t} d t \tilde{H}\right) \\
\tilde{H} & =V H V^{-1}+i \frac{\partial V}{\partial t} V^{-1}
\end{aligned}
$$

This factorization, so far, is just a mathematical identity. The idea now is to choose $V$ such that the interaction terms are eliminated in $\tilde{H}$. For the Abelian case, we can take the ansatz

$$
V=\exp \left[-i \int\left(e_{i}^{(c)} \hat{a}_{i}-a_{i}^{(c)} \hat{e}_{i}\right)\right]
$$

This leads to

$$
\begin{aligned}
\tilde{H}= & \frac{1}{2} \int\left(\hat{e}^{2}+\hat{b}^{2}\right)+\int\left[e_{i}^{(c)} \hat{e}_{i}+b_{i}^{(c)} \hat{b}_{i}+a_{i}^{(c)} J_{i}\right. \\
& \left.+\hat{a}_{i} J_{i}-\dot{a}_{i}^{(c)} \hat{e}_{i}+\dot{e}_{i}^{(c)} \hat{a}_{i}\right]+c \text {-number terms }
\end{aligned}
$$

where $b_{i}=\frac{1}{2} \epsilon_{i j k} \partial_{j} a_{k}$ is the magnetic field. We can write $\int b_{i}^{(c)} \hat{b}_{i}=-\int \nabla^{2} a_{i}^{(c)} \hat{a}_{i}$ via a partial integration. We then see that all the mixing terms can be eliminated by setting the coefficients of $\hat{e}_{i}$ and $\hat{a}_{i}$ in the bracketed terms to zero, i.e., by choosing $a_{i}^{(c)}$ and $e_{i}^{(c)}$ to be solutions of

$$
e_{i}^{(c)}-\dot{a}_{i}^{(c)}=0, \quad-\nabla^{2} a_{i}^{(c)}+\dot{e}_{i}^{(c)}+J_{i}=0
$$

These are the same equations as we had before for the background field. The splitting of $U$ as $V(t)^{-1} \tilde{U}(t, 0) V(0)$ shows that $V$ is the dressing factor, since the time-evolution given by $|\psi(t)\rangle=U(t, 0)|\psi(0)\rangle$ translates as

$$
[V(t)|\psi(t)\rangle]=\tilde{U}(t, 0)[V(0)|\psi(0)\rangle] .
$$

Thus, the dressed states $V|\psi\rangle$ evolve with the Hamiltonian $\tilde{H}$ in which the terms mixing $\left(\hat{a}_{i}, \hat{e}_{i}\right)$ and $\left(a_{i}^{(c)}, e_{i}^{(c)}\right)$ have been eliminated via (28). The remaining Hamiltonian shows that the soft photons behave as a decoupled free system. (The $c$-number terms are irrelevant for this discussion.)

A similar analysis can be done for the non-Abelian case, but it is trickier for two reasons: (1) There are gluon-gluon type interactions due to the cubic and quartic terms in the action or Hamiltonian. (2) The current $J_{i}^{A}=\dot{Z}_{i} Q^{A}$ is an operator (because of the $Q^{A}$ ) and hence the analogue of Eq. (28) will not yield $c$-number functions for $a_{i}^{(c)}, e_{i}^{(c)}$. We can parametrize $V$ in the form

$$
V=\exp \left[-i \int\left(e_{i}^{A(c)} \hat{a}_{i}^{A}-a_{i}^{A(c)} \hat{e}_{i}^{A}\right)+i K\right] .
$$

$\tilde{H}$, defined as in (25), will now contain many additional terms compared to (27) consisting of the gluon-gluon interaction terms, commutators between these and the exponent in $V$, and commutators of $Q_{A}$ with various terms including $a_{i}^{A(c)}$ and $e_{i}^{A(c)}$. A systematic perturbation series 
for $K$ in (30) can be written down although the calculation of the terms in the series can reveal infrared divergences. In the spirit of defining colored states as in perturbative QCD, if we neglect these additional corrections, the solutions will be similar to the Abelian case. Thus, to the lowest order,

$$
\hat{a}_{i}^{A}=\sum_{k} \frac{1}{\sqrt{2 \omega_{k} \mathcal{V}}}\left(c_{i}^{A}(k) e^{-i k x}+c_{i}^{A \dagger}(k) e^{i k x}\right)
$$

We can then write the dressing factor in (30) as $V=e^{\Phi}$, $\Phi=i \hat{\chi}^{A} Q_{A}$ with

$\hat{\chi}^{A}=-i \sum_{k} \frac{1}{\sqrt{2 \omega_{k} \mathcal{V}}}\left[\Omega_{i}^{+}(-k, p) c_{i}^{A}(k)-\Omega_{i}^{-}(k, p) c_{i}^{A^{\dagger}}(k)\right]$

In the fully interacting case, the expression for $V$ will be substantially more complicated, but we can still make the following general argument. $V$ depends on the canonical variables $\hat{e}_{i}^{A}, \hat{a}_{i}^{A}$ and on $Q_{A}$; the latter dependence is entirely from $\left(e^{(c)}\right)_{i}^{A}$ and $\left(a^{(c)}\right)_{i}^{A}$. All the variables appear in combinations such that all color indices are contracted to form invariants. As a result, we have the following property: A color rotation of $Q_{A}$ can be compensated by a color rotation of the operators $\hat{e}_{i}^{A}, \hat{a}_{i}^{A}$. Further, expectation values are evaluated by using commutation rules for $\hat{e}_{i}^{A}, \hat{a}_{i}^{A}$ which are invariant under color rotations. Writing $Q_{A}^{\prime}=$ $\mathcal{D}_{A B}(h) Q_{B}$, where $\mathcal{D}_{A B}(h)$ is the adjoint representation of a constant $x$-independent $h \in G$, these features tell us that

$$
V_{p}\left(Q_{A}^{\prime}, \hat{e}_{i}^{A}, \hat{a}_{i}^{A}\right)=V_{p}\left(Q_{A}, \hat{e}_{i}^{\prime A}, \hat{a}_{i}^{\prime A}\right)
$$

Here we have also indicated, via the subscript, the dependence of $V$ on the timelike vector $p_{\mu}$.

Consider now the overlap of two states given by

$$
I_{r s} \equiv\left\langle 0, r\left|V_{p}^{\dagger}\left(Q_{A}, \hat{e}_{i}^{A}, \hat{a}_{i}^{A}\right) V_{p^{\prime}}\left(Q_{A}, \hat{e}_{i}^{A}, \hat{a}_{i}^{A}\right)\right| 0, s\right\rangle .
$$

We insert $h h^{-1}$ between $V^{\dagger}$ and $V$ to write this as

$$
\begin{aligned}
I_{r s}= & \left\langle 0, r\left|V_{p}^{\dagger}\left(Q_{A}, \hat{e}_{i}^{A}, \hat{a}_{i}^{A}\right) h h^{-1} V_{p^{\prime}}\left(Q_{A}, \hat{e}_{i}^{A}, \hat{a}_{i}^{A}\right)\right| 0, s\right\rangle \\
= & \langle 0, r| h\left[h^{-1} V_{p}^{\dagger}\left(Q_{A}, \hat{e}_{i}^{A}, \hat{a}_{i}^{A}\right) h\right] \\
& \times\left[h^{-1} V_{p^{\prime}}\left(Q_{A}, \hat{e}_{i}^{A}, \hat{a}_{i}^{A}\right) h\right] h^{-1}|0, s\rangle \\
= & \left\langle 0, r\left|h V_{p}^{\dagger}\left(Q_{A}^{\prime}, \hat{e}_{i}^{A}, \hat{a}_{i}^{A}\right) V_{p^{\prime}}\left(Q_{A}^{\prime}, \hat{e}_{i}^{A}, \hat{a}_{i}^{A}\right) h^{-1}\right| 0, s\right\rangle \\
= & h_{r r^{\prime}} h_{s s^{\prime}}\left\langle 0, r^{\prime}\left|V_{p}^{\dagger}\left(Q_{A}^{\prime}, \hat{e}_{i}^{A}, \hat{a}_{i}^{A}\right) V_{p^{\prime}}\left(Q_{A}^{\prime}, \hat{e}_{i}^{A}, \hat{a}_{i}^{A}\right)\right| 0, s^{\prime}\right\rangle \\
= & h_{r r^{\prime}} h_{s s^{\prime}}\left\langle 0, r^{\prime}\left|V_{p}^{\dagger}\left(Q_{A}, \hat{e}_{i}^{A}, \hat{a}_{i}^{A}\right) V_{p^{\prime}}\left(Q_{A}, \hat{e}_{i}^{A}, \hat{a}_{i}^{A}\right)\right| 0, s^{\prime}\right\rangle \\
= & h_{r r^{\prime}} h_{s s^{\prime}} I_{r^{\prime} s^{\prime}} .
\end{aligned}
$$

This shows that $I_{r s}$ is an invariant tensor in the representation of the color charge algebra. As a result, we should have $I_{r s}=I \delta_{r s}$. This argument shows that the state of the color charge is not changed by the dressing factor. Timeevolution involved is given by the Hamiltonian $\tilde{H}$ which is invariant under color rotations and hence time-evolution also will not change the state of the color charge. This is a reflection of the superselection of color charge.

This result by itself is not sufficient to make any definite conclusion about the unitary implementation of color transformations. It is needed but is only part of the argument. We will now argue that any attempt to carry out a color rotation on the coherent states will run into problems. For this, consider the matrix element of a color rotation operator $h(\theta)=\exp \left(i \theta^{A} Q_{A}\right)$ between arbitrary dressed states given by

$$
M_{r s}=\left\langle 0, r\left|V^{\dagger}(Q, \hat{e}, \hat{a}) h(\theta) V(Q, \hat{e}, \hat{a})\right| 0, s\right\rangle .
$$

We will show, albeit to lowest order in perturbation theory (i.e., in the limit of neglecting self-interactions of the gluon), that this matrix element vanishes for any $h \neq 1$ as $\mu \rightarrow 0$, in a way similar to what happens for Lorentz transformations of $p_{\mu}$. This means that color transformations cannot be unitarily implemented on the dressed states in the sector with net nonzero color. A dressed state like $V(Q, \hat{e}, \hat{a})|0, s\rangle$ where $|0, s\rangle$ is the Fock vacuum for the infrared gluons, but can have charged particles characterized by the color index $s$ are precisely what we need as the charged states to be used in the lowest order in perturbation theory. So the implication of our result is that color transformations cannot be unitarily realized on the perturbative states.

To summarize, we have the non-Abelian generalization of the action (2) given in (21). The dressing factor, in the approximation of neglecting gluon-gluon interactions was obtained. We also obtained superselection of color charge states; this was obtained before in another way in [9]. And finally, we claim that the matrix element for a nontrivial color rotation on the coherent states is zero. The argument for the last statement, because it involves many technical points, is given below as a separate section.

\section{VANISHING MATRIX ELEMENTS FOR COLOR ROTATION}

Consider the matrix element of the color rotation in (36). Using $1=h h^{-1}$ to the left of $V^{\dagger}$, where $h$ is an element of the color group, we can write it as

$$
\begin{aligned}
M_{r s} & =\left\langle 0, r\left|h(\theta)\left[h^{-1}(\theta) V^{\dagger}(Q, \hat{e}, \hat{a}) h(\theta)\right] V(Q, \hat{e}, \hat{a})\right| 0, s\right\rangle \\
& =h_{r q}\left\langle 0, q\left|V^{\dagger}\left(Q^{\prime}, \hat{e}, \hat{a}\right) V(Q, \hat{e}, \hat{a})\right| 0, s\right\rangle,
\end{aligned}
$$

where $Q_{A}^{\prime}=h^{-1} Q_{A} h=\mathcal{D}_{A B}(h) Q_{B}$. We have chosen different color states $|r\rangle,|s\rangle$ for $M_{r s}$. Since

$$
V^{\dagger}\left(Q^{\prime}\right) V(Q)\left[V^{\dagger}\left(Q^{\prime}\right) V(Q)\right]^{\dagger}=1,
$$

$V^{\dagger}\left(Q^{\prime}\right) V(Q)$ is unitary and we see, by the Cauchy-Schwarz inequality, that the off-diagonal matrix element $\langle 0, q| V^{\dagger}\left(Q^{\prime}\right)$ $V(Q)|0, s\rangle$ does not exceed in magnitude the diagonal 
element $\left\langle 0, r\left|V^{\dagger}\left(Q^{\prime}\right) V(Q)\right| 0, r\right\rangle$. The vanishing of the latter is, thus, sufficient to show the vanishing of color rotation matrix element in (36), (37). So our aim is to show that the diagonal element $\left\langle 0, r\left|V^{\dagger}\left(Q^{\prime}\right) V(Q)\right| 0, r\right\rangle$ vanishes, for $Q^{\prime} \neq Q$, as $\mu \rightarrow 0$, in the limit of neglecting gluon-gluon interactions. ${ }^{1}$

However, establishing the vanishing of $\langle 0, r| V^{\dagger}\left(Q^{\prime}\right) \times$ $V(Q)|0, r\rangle$ is not so straightforward because, even if we pick $Q$ 's in an Abelian subalgebra, $Q_{A}^{\prime}$ can have components outside of this subalgebra leading to $\left[Q_{A}^{\prime}, Q_{B}\right] \neq 0$. Secondly, even though we can write $V=e^{\Phi}$ with $\Phi=$ $i \chi^{A} Q_{A}$ given as in (32), the normal ordering of this expression is also not straightforward since

$$
\left[Q_{A} c_{i}^{A}, Q_{B} c_{j}^{B^{\dagger}}\right]=Q^{2} \delta_{i j}+Q_{A} c_{j}^{B^{\dagger}}\left(-i f^{A B C}\right) c_{i}^{C},
$$

and the second term on the right-hand side can generate further commutator terms in reordering the series for $V$.

So, towards carrying out the calculation of the matrix element $\left\langle 0, r\left|V^{\dagger}\left(Q^{\prime}\right) V(Q)\right| 0, r\right\rangle$, we first consider

$$
\langle 0, r|V(Q)| 0, r\rangle=\left\langle 0, r\left|e^{i \hat{\chi}^{A} Q_{A}}\right| 0, r\right\rangle \equiv e^{-\frac{1}{2} W}
$$

This is the definition of $W$. By the general properties of such expectation values, we can write $W$ in terms of connected functions of $\hat{\chi}^{A} Q_{A}$. For this we note that $\hat{\chi}^{A}$ is a free bosonic field, although it has many components, so it has only connected two-point functions given by

$$
\left\langle\hat{\chi}^{A} \hat{\chi}^{B}\right\rangle=z \delta^{A B}
$$

where we use

$$
z=\left(\Omega^{+}, \Omega^{-}\right)=\frac{1}{16 \pi^{3}} \log (\lambda / \mu)(\vec{v}, \vec{v})
$$

This variable $z$ takes real values from zero to infinity, with $z \rightarrow \infty$ corresponding to $\mu \rightarrow 0$, so long as $(\vec{v}, \vec{v}) \neq 0$. ${ }^{2}$ Equation (41) shows that we can evaluate $W$ in terms of a series of Wick contractions on powers of $\chi$. It is easy to see that $W$ is real, with $W \geq 0$. It is also of the form

$$
W=2 Q^{2} z \sum_{0}^{\infty} w_{n}\left(\frac{z C_{\mathrm{ad}}}{2}\right)^{n}
$$

\footnotetext{
${ }^{1}$ For a complete proof of color breaking, we must also show that the above matrix element vanishes when $V(Q)$ is replaced by $\alpha V(Q)$ where $\alpha$ is any local observable. We will omit this proof here. An alternative proof of the result of this section is in [9].

${ }^{2}$ Later we use $z \rightarrow \infty$ to argue for the vanishing of the matrix elements for color rotations. Strictly speaking, this could be vitiated for $\vec{v}=0$. However, our result holds for any $\vec{v}$ of arbitrarily small but fixed magnitude.
}

where $w_{n}$ are numerical coefficients and $C_{\mathrm{ad}}$ is the quadratic Casimir for the adjoint representation of the group. We show these properties in the Appendix, where we also find

$$
w_{0}=\frac{1}{2}, \quad w_{1}=\frac{1}{4 !}, \quad w_{2}=\frac{5}{6 !} .
$$

Another property of importance to us is that, viewing $W$ as a function of $z$, it obeys the inequality

$$
W(z) \geq W\left(z_{1}\right), \quad z \geq z_{1} .
$$

The reasoning behind this result is that, for $z>z_{1}$, more states are included because we are increasing the range of $\vec{k}$-values and hence we should expect some such condition following from the completeness relation. More specifically, the required expectation value can be written as

$$
\begin{aligned}
\left\langle e^{\Phi}\right\rangle & =\left\langle e^{i \hat{\chi} \cdot Q}\right\rangle=\left\langle e^{-i \hat{\chi} \cdot Q}\right\rangle=\langle\cos \hat{\chi} \cdot Q\rangle \\
& =\left\langle 1-2 \sin ^{2}(\hat{\chi} \cdot Q / 2)\right\rangle
\end{aligned}
$$

Here we have used the fact that only even powers of $\hat{\chi}$ can contribute and that the trigonometric relations hold as operator statements since each expression is defined by the power series expansion. From (46), $2\left\langle\sin ^{2}(\hat{\chi} \cdot Q / 2)\right\rangle=$ $1-e^{-W / 2}$. We now have

$$
\begin{aligned}
\left\langle\sin ^{2}\left(\frac{\hat{\chi} \cdot Q}{2}\right)\right\rangle_{z}= & \sum_{\alpha}\left\langle 0, r\left|\sin \left(\frac{\hat{\chi} \cdot Q}{2}\right)\right| \alpha, s\right\rangle \\
& \times\left\langle\alpha, s\left|\sin \left(\frac{\hat{\chi} \cdot Q}{2}\right)\right| 0, r\right\rangle \\
= & \left\langle\sin ^{2}\left(\frac{\hat{\chi} \cdot Q}{2}\right)\right\rangle_{z_{1}} \\
& +\sum_{\alpha^{\prime}}\left|\left\langle 0, r\left|\sin \left(\frac{\hat{\chi} \cdot Q}{2}\right)\right| \alpha^{\prime}, s\right\rangle\right|^{2} \\
\geq & \left\langle\sin ^{2}\left(\frac{\hat{\chi} \cdot Q}{2}\right)\right\rangle_{z_{1}}
\end{aligned}
$$

In this equation $\alpha^{\prime}$ indicates states which involve at least one value of $\vec{k}$ beyond the range given by $z_{1}$. Using the inequality (47) in the expression for $W$, we find the result (45).

The property (45) of $W$ shows that its derivative with respect to $z$ is non-negative. (In fact, the derivative will be positive since we obtain $W(z)=W\left(z_{1}\right)$ only if there are no states with $\vec{k}$ beyond the range given by $z_{1}$.) The next property we need is that $W(z)$ does not saturate to a finite value as $z \rightarrow \infty$. In fact

$$
W(z) \rightarrow \infty \quad \text { as } \quad z \rightarrow \infty
$$


For showing this result, we express $W$ as an integral. As mentioned earlier, $\hat{\chi}^{A}$ is a free bosonic field, and hence the only connected correlators are the two-point ones. Therefore we can represent it using a Gaussian integral, i.e.,

$$
\begin{aligned}
\left\langle 0, r\left|e^{i \hat{\chi} \cdot Q}\right| 0, r\right\rangle= & \frac{1}{(2 \pi z)^{\operatorname{dim} G / 2}} \int[d \sigma] \\
& \times \exp \left(-\frac{1}{2 z} \sigma^{A} \sigma^{A}\right)\left\langle r\left|e^{i \sigma \cdot Q}\right| r\right\rangle .
\end{aligned}
$$

(The $\sigma$ 's are $c$-number variables.) This is a finite dimensional integral, there are $\operatorname{dim} G$ independent variables $\chi^{A}$ which are real. The quantity $\left\langle r\left|e^{i \sigma \cdot Q}\right| r\right\rangle$ in the integrand is a function of the $\sigma$ 's; it is the diagonal matrix element $g_{r r}$ of the group element $g=\exp (i \sigma \cdot Q)$ in the representation corresponding to the states $|r\rangle$. However, it should be kept in mind that the integration is not over the group volume (i.e., not with the Haar measure), but over all real values of the group parameters, $\sigma^{A}$ in this case. Now, $\left\langle r\left|e^{i \sigma \cdot Q}\right| r\right\rangle$ must be a periodic function of the $\sigma$ 's and so it can be expanded in a Fourier series as

$$
\left\langle r\left|e^{i \sigma \cdot Q}\right| r\right\rangle=\sum_{\{n\}} C_{n_{1}, n_{2}, \ldots, n_{d}} \sin \left(\frac{n_{1} \sigma_{1}}{l_{1}}\right) \cdots \sin \left(\frac{n_{d} \sigma_{d}}{l_{d}}\right)+\cdots,
$$

where $d=\operatorname{dim} G$ and the ellipsis indicates terms with cosines and mixtures of sines and cosines. We may not have the same period for all $\sigma$ 's; that will depend on the normalization of the generators, so we include parameters $l_{1}, \ldots, l_{d}$. The key point is that, with the Gaussian measure (49), the average of any periodic function goes to zero as $z \rightarrow \infty$ as seen from

$$
\begin{aligned}
& \left\langle\sin \left(\frac{n_{1} \sigma_{1}}{l_{1}}\right)\right\rangle \\
& \quad=\frac{1}{2 i}\left[\exp \left(-n_{1}^{2} z / l_{1}^{2}\right)-\text { complex conjugate }\right] \underset{z \rightarrow \infty}{\longrightarrow} 0 \\
& \left\langle\cos \left(\frac{n_{1} \sigma_{1}}{l_{1}}\right)\right\rangle \\
& \quad=\frac{1}{2}\left[\exp \left(-n_{1}^{2} z / l_{1}^{2}\right)+\text { complex conjugate } \underset{z \rightarrow \infty}{\longrightarrow} 0\right.
\end{aligned}
$$

This is equivalent to the statement in (48).

Finally we come to the matrix element of interest, namely, $\left\langle 0, r\left|V^{\dagger}\left(Q^{\prime}\right) V(Q)\right| 0, r\right\rangle$, with $Q^{\prime}=h^{-1} Q h \neq Q$. From the Cauchy-Schwarz inequality, this obeys

$$
\begin{aligned}
\left|\left\langle 0, r\left|V^{\dagger}\left(Q^{\prime}\right) V(Q)\right| 0, r\right\rangle\right|^{2} \leq & \left\langle 0, r\left|V^{\dagger}\left(Q^{\prime}\right) V\left(Q^{\prime}\right)\right| 0, r\right\rangle \\
& \times\left\langle 0, r\left|V^{\dagger}(Q) V(Q)\right| 0, r\right\rangle .
\end{aligned}
$$

Since the right-hand side is 1 , we have

$$
\left|\left\langle 0, r\left|V^{\dagger}\left(Q^{\prime}\right) V(Q)\right| 0, r\right\rangle\right|^{2} \leq 1 .
$$

Equality is obtained only for $Q^{\prime}=Q$, i.e., for $h=1$. We can now write this matrix element also as an integral over $\sigma$ 's,

$$
\begin{aligned}
\left\langle 0, r\left|e^{-i \hat{\varkappa} \cdot Q^{\prime}} e^{i \hat{\varkappa} \cdot Q}\right| 0, r\right\rangle= & \frac{1}{(2 \pi z)^{\operatorname{dim} G / 2}} \int[d \sigma] \\
& \times \exp \left(-\frac{1}{2 z} \sigma^{A} \sigma^{A}\right)\left\langle r\left|e^{-i \sigma \cdot Q^{\prime}} e^{i \sigma \cdot Q}\right| r\right\rangle .
\end{aligned}
$$

Using $Q_{A}^{\prime}=h^{-1} Q_{A} h$,

$$
\left\langle r\left|e^{-i \sigma \cdot Q^{\prime}} e^{i \sigma \cdot Q}\right| r\right\rangle=h_{r s}^{-1} h_{p q}\left\langle s\left|e^{-i \sigma \cdot Q}\right| p\right\rangle\left\langle q\left|e^{i \sigma \cdot Q}\right| r\right\rangle .
$$

Once again, the product $\left\langle s\left|e^{-i \sigma \cdot Q}\right| p\right\rangle\left\langle q\left|e^{i \sigma \cdot Q}\right| r\right\rangle$ is a periodic function of the $\chi$ 's, not equal to 1 for all $\sigma$. In fact, it is easy to see that this is not identically 1 by considering $h$ near the identity. Being periodic, it can be expanded in a Fourier series and by the result (51), the average matrix element in (54) will give zero. This proves the vanishing of the matrix element $\left\langle 0, r\left|e^{-i \hat{\chi} \cdot Q^{\prime}} e^{i \hat{\chi} \cdot Q}\right| 0, r\right\rangle$. In turn, this shows that the matrix elements of the color rotation in (37) will vanish for any $h \neq 1$, as $z \rightarrow \infty$, or as $\mu \rightarrow 0$. And finally, this leads to the statement given after (36) about the problem with a unitary realization of color transformations in the sector with net nonzero color.

\section{THE PROBLEM OF DEFINING COLOR CONFINEMENT}

In this paper, we have analyzed some of the infrared issues in a gauge theory in terms of a particular splitting of the action into a part for the hard modes and a part for the soft modes, with a diffeomorphism for the hard part. This diffeomorphism which can be viewed in terms of the lapse and shift functions for the metric encodes the overall motion of the collection of charged particles. These functions also characterize the timelike vector which defines the dressing of the Fock states for the soft fields and hence the coherent states of soft modes to be used in evaluating amplitudes. Our results reproduce in a novel way the known results regarding the spontaneous breaking of Lorentz symmetry in the charged sectors of quantum electrodynamics.

We have also analyzed the question of a spontaneous breaking of color transformations in a non-Abelian gauge theory. Our basic result is that the matrix elements for color rotations cannot be unitarily implemented (for states of nonzero color charge) if the following premises hold:

(1) Perturbation theory can be used and gluon-gluon interactions can be neglected as a lowest order approximation. (Theories where such an approximation can be valid would include those with sufficient 
number of matter fields so that asymptotic freedom is not obtained or those which admit a non-Abelian Coulomb phase.)

(2) The velocity $v_{i}=p_{i} / p_{0}$ is not zero. Here, $p_{\mu}$ is the timelike vector used to define the coherent states for the soft modes of the gauge boson. ( $v_{i}$ of arbitrarily small magnitudes are adequate for the argument, so this is presumably not a serious restriction.)

(3) We take $\mu \rightarrow 0$ at fixed $\lambda$; eventually the latter can be taken to be zero as well. (This is the same situation as for showing the spontaneous breaking of Lorentz symmetry in electrodynamics.)

(4) The gauge bosons are massless.

In this context, we also refer to $[8,9]$ where arguments based on non-Abelian superselection rules are presented to show that non-Abelian gauge groups are spontaneously broken.

Our results do not directly impact standard perturbative QCD since we do have asymptotic freedom in this case, and so one cannot justify the use of pertrurbation theory or premise 4 above, as the we take $\mu \rightarrow 0$. Also, for QCD calculations, one can restrict oneself to states with total charge equal to zero, i.e., to states which are QCD singlets.

Our analysis does show that defining asymptotically nonfree non-Abelian theories or non-Abelian Coulomb phases can be problematic. It also calls into question the definition of color confinement. The standard lore has been that we should implement the Gauss law with test functions which vanish at spatial infinity on physical states. This would still allow for charged states which transform as some representation of the color group. Thus, a priori there is no obstruction to defining color transformations on the single particle states. Confinement is then the statement that, for dynamical reasons, the spectrum of the Hamiltonian does not contain the charged states. One can use, in some cases where there are no fields transforming according to the fundamental representation, the expectation value of the Wilson loop as a diagnostic for confinement. This is the more conventional signature one looks for in, say, lattice simulations.

However, our results go further. If, as we have argued, color transformations cannot be defined on perturbative gluon states, the theory presumably evades one or more of the premises to our argument, listed above. One possibility is that there cannot be gluon states of arbitrarily low energy (so that we cannot take $z \rightarrow \infty$.) It could also be that single charged excitations carry infinite energy. Another possibility is that one can have configurations of zero total charge without the cluster property so that single charged states cannot be separated off.

These arguments clearly indicate that our analysis has implications for the mass gap and confinement issues in the non-Abelian theory. It does raise a somewhat finely nuanced problem about color rotations. While we are not able, at this stage, to give a definite answer to what happens in theories of the type we are considering, it is clear that further analysis is very important.

\section{ACKNOWLEDGMENTS}

This research was supported in part by the U.S. National Science Foundation Grant No. PHY-1519449 and by PSCCUNY awards.

\section{APPENDIX A: REMARKS ON THE CANONICAL FRAMEWORK FOR $Z^{\mu}$ IN (2)}

In this Appendix, we will work out some general features of the action (2). We will also add a Dirac action as the matter part. The induced metric has frame fields given by $\mathcal{E}^{0}=\dot{Z}_{0} d t, \mathcal{E}^{i}=d x^{i}+\dot{Z}^{i} d t$. These obey $d \mathcal{E}^{a}=0$, so that the spin connection may be taken as zero. Thus, the Dirac action has the form

$$
\begin{aligned}
S_{\text {Dirac }} & =\int d^{4} x \sqrt{-g} \bar{\psi}\left[i\left(\mathcal{E}^{-1}\right)_{a}^{\mu} \gamma^{a} D_{\mu}-m\right] \psi \\
& =\int d^{4} x \dot{Z}_{0} \bar{\psi}\left[i \frac{\gamma^{0}}{\dot{Z}_{0}}\left(D_{0}-\dot{Z}^{i} D_{i}\right)+i \gamma^{i} D_{i}-m\right] \psi
\end{aligned}
$$

The electromagnetic part of the action $S_{1}$ simplifies as

$$
\begin{aligned}
S_{1 \mathrm{em}}= & -\frac{1}{4} \int d^{4} x\left[-2 \frac{E^{2}}{\dot{Z}_{0}}+2 \dot{Z}_{0} B^{2}+4 \frac{\dot{Z}_{i}(\vec{E} \times \vec{B})_{i}}{\dot{Z}_{0}}\right. \\
& \left.-2 \frac{\left(\dot{Z}_{i} \dot{Z}_{i} B^{2}-\left(\dot{Z}_{i} B_{i}\right)^{2}\right)}{\dot{Z}_{0}}\right] .
\end{aligned}
$$

Notice that $v_{i} \equiv \dot{Z}_{i} / \dot{Z}_{0}$ is independent of the spatial coordinates, so that we have $\int d^{3} x \dot{Z}_{i}(\vec{E} \times \vec{B})_{i} / \dot{Z}_{0}=\dot{Z}_{i} /$ $\dot{Z}_{0} \int d^{3} x,(\vec{E} \times \vec{B})_{i}=0$, by virtue of rotational invariance. This will remove one of the terms in (A2). Further, we have invariance under reparametrization of the variable $t$; The action can be written entirely in terms of $Z_{0}$ and the three spatial coordinates using $d^{4} x \dot{Z}_{0}=d^{3} x d Z_{0}$, etc. This invariance will lead to a zero Hamiltonian for the evolution with respect to $t$. The evolution with respect to $x^{0}$ is what is relevant; equivalently, we can also make the gauge choice $\dot{Z}_{0}=1$. In this case,

$$
\begin{aligned}
S_{1 \mathrm{em}} & =\int d^{4} x\left[\frac{1}{2}\left(E^{2}-B^{2}\right)+\frac{1}{2}\left(v^{2} B^{2}-(\vec{v} \cdot \vec{B})^{2}\right)\right] \\
S_{\text {Dirac }} & =\int d^{4} x \bar{\psi}\left[i \gamma^{0} \partial_{0}-i \gamma^{0} \vec{v} \cdot \vec{D}+i \gamma^{i} D_{i}-m\right] \psi .
\end{aligned}
$$

For the infrared modes, we have

$$
S_{2}+S_{3}=\frac{1}{2} \int\left(e^{2}-b^{2}\right)-q \int a_{i} v^{i}
$$

where $e_{i}=\partial_{0} a_{i}, b_{i}=(\nabla \times a)_{i}$. We have also chosen the $A_{0}=a_{0}=0$ gauge. The Hamiltonian can now be worked out as 


$$
\begin{aligned}
H= & \int\left[\frac{1}{2}\left(E^{2}+B^{2}\right)+\frac{1}{2}\left(v^{2} B^{2}-(\vec{v} \cdot \vec{B})^{2}\right)\right. \\
& \left.-\bar{\psi}\left(i \gamma^{i} D_{i}-m\right) \psi+\frac{1}{2}\left(e^{2}+b^{2}\right)\right] .
\end{aligned}
$$

This is manifestly positive, except for the Dirac term, which, as usual, is not positive. Upon quantization, the Dirac Hamiltonian will also be positive after the usual redefinition of the negative energy states.

It is also useful to look at the dynamics of $Z^{i}$. Assuming that $\int B^{2}$ is small, or that we can neglect it as a first approximation, we see that

$$
\frac{\partial L}{\partial v^{i}}=\int \psi^{\dagger}\left(-i D_{i} \psi\right)-q a_{i}(Z) \equiv P_{i}
$$

Notice that we cannot solve this for $v^{i}$ in terms of the momentum $P_{i}$. From the equation of motion for $Z^{i}$, we get

$$
\frac{d}{d t} \int \psi^{\dagger}\left(-i D_{i} \psi\right)=q\left(e_{i}+f_{j i} \dot{Z}^{j}\right)
$$

As we show in Appendix B, the right-hand side of this equation vanishes, showing that $\int \psi^{\dagger}\left(-i D_{i} \psi\right)$ is independent of time. This is the total momentum of the particle system. (If we had kept the contribution for the $B_{i}\left(v^{2} \delta^{i j}-v^{i} v^{j}\right) B_{j}$-term in the action, there would be some correction to the total momentum; qualitative features will not be changed.) To relate $P_{i}$ to $v^{i}$, we have to use the Hamiltonian. Since $P_{i}$ is conjugate to $Z^{i}$, we get

$$
\left[Z^{i}, P_{j}\right]=i \delta_{j}^{i}, \quad i \dot{Z}^{i}=\left[Z^{i}, H\right] .
$$

Thus, if the Hamiltonian is expressed using $P_{i}$ (among other variables) for some charged particle state, then (A8) can relate $\dot{Z}^{i}$ and $P_{i}$.

\section{APPENDIX B: THE CLASSICAL INFRARED FIELDS}

We start by calculating the solution of the equation of motion for $a_{\mu}$ which is needed to evaluate the factor $f^{\mu \nu} J_{\nu}$ used in Sec. III. This solution is also what is designated as $a_{\mu}^{(c)}$ in Sec. IV. The source current is given by

$$
\begin{aligned}
J_{\mu} & =q \int d \tau \frac{d Z_{\mu}}{d \tau} \delta^{(4)}(y-Z(\tau)) \\
& =q \int d \tau p_{\mu} \delta^{(4)}(y-Z(0)-p \tau),
\end{aligned}
$$

where $p_{\mu}=d Z_{\mu} / d \tau$. The solution in the gauge $a_{0}=0$, $\nabla \cdot a=0$ is given by

$$
\begin{aligned}
a_{i}^{(c)} & =-q \int d \tau \frac{d^{4} k}{(2 \pi)^{4}}\left(p_{i}-k_{i} \frac{\vec{k} \cdot \vec{p}}{\vec{k}^{2}}\right) \frac{1}{k^{2}+i \epsilon} e^{-i k(x-Z(0)-p \tau)} \\
e_{i}^{(c)} & =q \int d \tau \frac{d^{4} k}{(2 \pi)^{4}}\left(p_{i}-k_{i} \frac{\vec{k} \cdot \vec{p}}{\vec{k}^{2}}\right) \frac{i k_{0}}{k^{2}+i \epsilon} e^{-i k(x-Z(0)-p \tau)}
\end{aligned}
$$

We have used the time-ordered (or Feynman) Green's function as this will be what is relevant in the functional integral in Sec. IV. The electric field is given as $e_{i}^{(c)}=\dot{a}_{i}^{(c)}$. In $f^{\mu \nu} J_{\nu}$, first consider the term

$$
f^{0 i} J_{i}=e_{i}^{(c)} J_{i}=\int d \tau e_{i}^{(c)}(Z) p_{i} \delta^{(4)}(x-Z(\tau)) .
$$

$Z^{\mu}(\tau)$ is the world line corresponding to the current or the overall motion of the droplet. In (B3), the field $e_{i}^{(c)}$ is evaluated on this world line. Taking account of the $i \epsilon$ prescription,

$$
\begin{aligned}
e^{(c)}(Z)_{i}= & \frac{q}{2} \int \frac{d^{3} k}{(2 \pi)^{3}}\left(p_{i}-k_{i} \frac{\vec{k} \cdot \vec{p}}{\vec{k}^{2}}\right) \\
& \times\left[\int_{-\infty}^{\tau} d \tau^{\prime} e^{-i k p\left(\tau-\tau^{\prime}\right)}-\int_{\tau}^{\infty} d \tau^{\prime} e^{i k p\left(\tau-\tau^{\prime}\right)}\right] .
\end{aligned}
$$

In the first of the $\tau$-integrals, we change variables to $u=$ $\tau^{\prime}-\tau$ and in the second to $u=\tau-\tau^{\prime}$ to get

$$
\begin{gathered}
\int_{-\infty}^{\tau} d \tau^{\prime} e^{-i k p\left(\tau-\tau^{\prime}\right)}-\int_{\tau}^{\infty} d \tau^{\prime} e^{i k p\left(\tau-\tau^{\prime}\right)} \\
=\int_{-\infty}^{0} d u e^{i k p u}-\int_{-\infty}^{0} d u e^{i k p u}=0 .
\end{gathered}
$$

This shows that the electric field evaluated on the world line itself is zero. Consider now the magnetic field which contributes to $f^{i j} J_{j}$. Evaluating it on the world line we find

$\epsilon_{i a b} \partial_{a} a_{b}^{(c)}=(-i q) \epsilon_{i a b} p_{b} \int d \tau^{\prime} \frac{d^{4} k}{(2 \pi)^{4}} \frac{k_{a}}{k^{2}+i \epsilon} e^{-i k p\left(\tau-\tau^{\prime}\right)}$

Other than $k_{a}$, the only vector we have in this expression is $p_{a}$. The integral, evaluated with rotationally invariant cutoffs $\lambda, \mu$, will, thus, be proportional to $p_{a}$ and hence the expression in (B6) will vanish. This confirms the assertion at the end of Sec. III that the dissipation effect due to the soft photons will vanish.

There is another way to write down the solution which will be useful for simplifying $V$. For this, without loss of generality, we can take $Z^{\mu}(0)$, the origin of the world line of the droplet, to be zero. In the gauge $\partial_{\mu} a^{\mu}=0$, the solution is given by 


$$
\begin{aligned}
a_{\nu}= & -\int d^{4} y \frac{d^{4} k}{(2 \pi)^{4}} \frac{1}{k^{2}+i \epsilon} e^{-i k(x-y)} J_{\nu}(y) \\
= & i \int d^{4} y \frac{d^{3} k}{(2 \pi)^{3}} \frac{1}{2 \omega_{k}}\left[\theta\left(x^{0}-y^{0}\right) e^{-i \omega\left(x^{0}-y^{0}\right)+\vec{k} \cdot(\vec{x}-\vec{y})}\right. \\
& \left.-\theta\left(y^{0}-x^{0}\right) e^{i \omega\left(x^{0}-y^{0}\right)-\vec{k} \cdot(\vec{x}-\vec{y})}\right] J_{\nu}(y) .
\end{aligned}
$$

Carrying out the $y^{0}$-integration, we find, for $x^{0}>y^{0}$,

$$
a_{\nu}=q p_{\nu} \int \frac{d^{3} k}{(2 \pi)^{3}} \frac{1}{2 \omega_{k}} \frac{1}{p k-i \epsilon} e^{i \vec{k} \cdot \tilde{x}},
$$

where $p k=p_{0} \omega_{k}-\vec{p} \cdot \vec{k}$ and $\tilde{\vec{x}}=\vec{x}-\left(\vec{p} x^{0}\right) / p_{0}$. The contribution for $y^{0}>x^{0}$ is similar except that we have $p k+i \epsilon$ in the denominator and the exponential becomes $e^{-\vec{i} \cdot \vec{x}}$. Combining the two and transforming to the gauge $a_{0}=0$ and $\partial_{i} a_{i}=0$, we get

$$
a_{i}=q \int \frac{d^{3} k}{(2 \pi)^{3}} \frac{1}{2 \omega_{k}}\left(\Omega_{i}^{+} e^{-\overrightarrow{i k} \cdot \tilde{x}}+\Omega_{i}^{-} e^{i \vec{k} \cdot \tilde{x}}\right),
$$

where

$$
\Omega_{i}^{ \pm}(k, p)=q\left(p_{i}-k_{i} \frac{\vec{k} \cdot \vec{p}}{\vec{k}^{2}}\right) \frac{1}{p k \pm i \epsilon}
$$

The corresponding electric field is given by

$$
e_{i}=q \int \frac{d^{3} k}{(2 \pi)^{3}} \frac{i \omega_{k}}{2 \omega_{k}}\left(\Omega_{i}^{+} e^{-i \vec{k} \cdot \tilde{x}}-\Omega_{i}^{-} e^{\vec{i} \cdot \tilde{x}}\right) .
$$

The solutions (B9) and (B11) agree with the results (B2). To see this one needs to carry out the integrations over $k_{0}$ and $\tau$ in (B2).

\section{APPENDIX C: PROPERTIES OF EXPECTATION VALUE FOR DRESSING FACTOR}

The function $W$ was defined in (40) by

$$
\langle 0, r|V(Q)| 0, r\rangle=\left\langle 0, r\left|e^{\Phi}\right| 0, r\right\rangle=\left\langle 0, r\left|e^{i \hat{\chi}^{A} Q_{A}}\right| 0, r\right\rangle \equiv e^{-\frac{1}{2} W} .
$$

Here $\Phi=i \hat{\chi}^{A} Q_{A}$. We will now show some of the properties of $W$ used in Sec. V.

Property 1: $\mathrm{W}$ is real and $e^{-\frac{1}{2} W} \geq 0$. This follows from the fact that we need even powers of $\hat{\chi}$ to obtain a nonzero Wick contraction.

Property 2: $W$ is positive semidefinite. The CauchySchwarz inequality for states $|A\rangle=e^{\Phi}|0\rangle$ and $|B\rangle=|0\rangle$ and the fact that $\left(e^{\Phi}\right)^{\dagger}=e^{-\Phi}$, give the result $\left|\left\langle e^{\Phi}\right\rangle\right|^{2} \leq\left\langle 0\left|e^{-\Phi} e^{\Phi}\right| 0\right\rangle$, which implies $\left|\left\langle e^{\Phi}\right\rangle\right| \leq 1$; this shows that $W \geq 0$. This is important in reconciling (45) and (48).
Property 3: $W$ has the form

$$
W=2 Q^{2} z \sum_{0}^{\infty} w_{n}\left(\frac{z C_{\mathrm{ad}}}{2}\right)^{n}
$$

Thus, apart from the overall $Q^{2}$, the rest of the terms have the combination $z C_{\text {ad }} / 2$. This result can be seen as follows. First of all we carry out a scaling $\Omega^{ \pm} \rightarrow a \Omega^{ \pm}, Q_{A} \rightarrow Q_{A} / a$ for some real parameter $a \neq 0$. Under this change, $\Phi \rightarrow \Phi$, so $W$, defined in terms of $\Phi$ as in (C1), is unchanged. The scaling is equivalent to $z \rightarrow a^{2} z, Q^{2} \rightarrow Q^{2} / a^{2}$. The commutations rules for the $Q$ 's become

$$
\left[\frac{Q_{A}}{a}, \frac{Q_{B}}{a}\right]=i \frac{f_{A B C}}{a} \frac{Q_{C}}{a} .
$$

Thus, under the scaling, we must have $f_{A B C} \rightarrow \frac{f_{A B C}}{a}$ and $C_{\text {ad }} \rightarrow C_{\text {ad }} / a^{2}$. Therefore, the invariant combinations are $Q^{2} z$ and $z C_{\text {ad }} / 2$, and $W$ must be a function of these.

Secondly, consider calculating $\left\langle e^{\Phi}\right\rangle$ by expansion of the exponential for representations which are of large dimensions compared to the adjoint representation,

$$
\left\langle e^{\Phi}\right\rangle=\sum_{n} \frac{1}{(2 n) !}\langle\Phi \Phi \cdots \Phi\rangle .
$$

After contracting the $c^{A}, c^{A \dagger}$ in $\Phi$, i.e., after using (32) and (41), we get products like $\left\langle Q_{A} Q_{B} \cdots\right\rangle$. The highest power in this corresponds to $\left(Q^{2}\right)^{n}$ which is obtained by neglecting the commutators. This contribution exponentiates to $\exp \left[\frac{1}{2}\langle\Phi \Phi\rangle_{c}\right]$, where the subscript $c$ indicates the connected component. For large representations, the commutators are subdominant. (This can also be seen by writing the product of the $Q$ 's using star products.) This fact shows that higher powers of $Q^{2}$ are excluded from $W$. This can be seen by examining the behavior of a sample of two terms in the expansion for $W$; the latter is of the form

$$
\frac{W}{2}=\frac{1}{2}\langle\Phi \Phi\rangle_{c}+\frac{1}{4 !}\langle\Phi \Phi \Phi \Phi\rangle_{c}+\cdots,
$$

where, again, the subscript $c$ denotes the connected component. In $\left\langle e^{\Phi}\right\rangle$, the term with $6 \Phi$ 's is, thus,

$\left\langle e^{\Phi}\right\rangle \sim \frac{1}{3 !}\left(\frac{1}{2}\langle\Phi \Phi\rangle_{c}\right)^{3}+\left(\frac{1}{2}\langle\Phi \Phi\rangle_{c}\right) \frac{1}{4 !}\langle\Phi \Phi \Phi \Phi\rangle_{c}+\cdots$

The right-hand side corresponds to the 6-point function for $\Phi$ 's, which should be of order $\left(Q^{2}\right)^{3}$. This is already accounted for by the $\left(\frac{1}{2}\langle\Phi \Phi\rangle_{c}\right)^{3}$ term. Thus, if $\langle\Phi \Phi \Phi \Phi\rangle_{c}$ is of any order higher than $Q^{2}$, we would have a contradiction. This shows that we can have only one overall power of $Q^{2}$ in $W$. Thus, of the two invariants $Q^{2} z$ and $z C_{\text {ad }} / 2$, we can have only one power of $Q^{2} z$ in $W$. The conclusion is that we do obtain the result $(\mathrm{C} 2)$. 
It is instructive to see how this works out for the first few terms of the expansion of $\left\langle e^{\Phi}\right\rangle$. We can write this expectation value out as

$$
\left\langle e^{\Phi}\right\rangle=1+\frac{1}{2}\langle\Phi \Phi\rangle+\frac{1}{4 !}\langle\Phi \Phi \Phi \Phi\rangle+\cdots
$$

For the term with two $\Phi$ 's, we get

$$
\langle\Phi \Phi\rangle=-\left\langle\hat{\chi}^{A} \hat{\chi}^{B}\right\rangle\left\langle Q_{A} Q_{B}\right\rangle=-z\left\langle Q^{2}\right\rangle .
$$

For the next term, we have via the Wick contractions

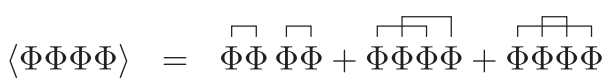

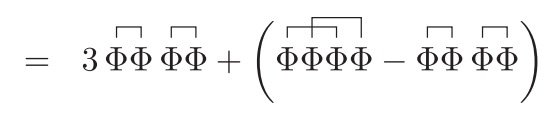

The connected part of the 4-point function is, thus, given by

$$
\begin{aligned}
\overparen{\Phi \Phi \Phi \Phi}-\Phi \Phi \Phi \Phi & =z^{2}\left\langle\left[Q_{A} Q_{B} Q_{A} Q_{B}-Q_{A} Q_{A} Q_{B} Q_{B}\right]\right\rangle \\
& =-\frac{C_{\mathrm{ad}}}{2} Q^{2} z^{2}=-Q^{2} z\left(\frac{z C_{\mathrm{ad}}}{2}\right)
\end{aligned}
$$

Notice that there is only one power of $Q^{2} z$ here. Proceeding in this way to the next order, we find

$$
\begin{aligned}
\left\langle e^{\Phi}\right\rangle= & e^{-W / 2}=\exp \left[-Q^{2} z\left(\frac{1}{2}+\frac{1}{4 !}\left(z C_{\mathrm{ad}} / 2\right)\right.\right. \\
& \left.\left.+\frac{5}{6 !}\left(z C_{\mathrm{ad}} / 2\right)^{2}+\cdots\right)\right] .
\end{aligned}
$$

[1] F. Bloch and A. Nordsieck, Phys. Rev. D 52, 54 (1937); P. A. M. Dirac, Can. J. Phys. 33, 650 (1955); D. R. Yennie, S. C. Frautschi, and H. Suura, Ann. Phys. (N.Y.) 13, 379 (1961); J. D. Dollard, J. Math. Phys. 5, 729 (1964); S. Weinberg, Phys. Rev. D 140, B516 (1965).

[2] S. Weinberg, The Quantum Theory of Fields: Volume I Foundations (Cambridge University Press, Cambridge, England, 1995).

[3] V. Chung, Phys. Rev. D 140, B1110 (1965); T. W. B. Kibble, J. Math. Phys. 9, 315 (1968); Phys. Rev. D 173, 1527 (1968); 174, 1882 (1968); 175, 1624 (1968); in Lectures in Theoretical Physics, edited by K. T. Mahanthappa and W. E. Brittin, Vol. 11D (Gordon and Breach, 1969); G. Roepstorff, Commun. Math. Phys. 19, 301 (1970).

[4] P. P. Kulish and L. D. Faddeev, Teor. Mat. Fiz. 4, 153 (1970) [Theor. Math. Phys. 4, 745 (1971)]; D. Zwanziger, Phys. Rev. D 11, 3481 (1975).

[5] T. He, P. Mitra, A. P. Porfyriadis, and A. Strominger, J. High Energy Phys. 10 (2014) 112; M. Campiglia and A. Laddha, J. High Energy Phys. 07 (2015) 115; D. Kapec, M. Pate, and A. Strominger, Adv. Theor. Math. Phys. 21, 1769 (2017).

[6] D. Buchholz, Commun. Math. Phys. 85, 49 (1982); Phys. Lett. B 174, 331 (1986).

[7] J. Fröhlich, G. Morchio, and F. Strocchi, Ann. Phys. (N.Y.) 119, 241 (1979); Phys. Lett. B 89, 61 (1979); J. Fröhlich, Commun. Math. Phys. 66, 223 (1979).

[8] A. P. Balachandran and S. Vaidya, Eur. Phys. J. Plus 128, 118 (2013); A. P. Balachandran, S. Kurkcuoglu, A. R. de Queiroz, and S. Vaidya, Eur. Phys. J. C 75, 89 (2015).

[9] A. P. Balachandran, Mod. Phys. Lett. A 31, 1650060 (2016).

[10] D. Carney et al., arXiv:1803.02370.

[11] R. Arnowitte, S. Deser, and C. Misner, Phys. Rev. D 116, 1322 (1959); P. A. M. Dirac, Lectures on Quantum Mechanics (Yeshiva University, NY, 1964); K. V. Kuchar, J. Math. Phys. 17, 801 (1976); C. G. Torres, J. Math. Phys.
33, 3802 (1992); T. Andrade, D. Marolf, and C. Deffayet, Classical Quantm Gravity 28, 105002 (2011).

[12] A. Ashtekar and R. O. Hansen, J. Math. Phys. 19, 1542 (1978); A. Ashtekar, Phys. Rev. Lett. 46, 573 (1981); A. Ashtekar and M. Streubel, Proc. Roy. Soc. A 376, 585 (1981); A. Ashtekar, Asymptotic Quantization: Based On 1984 Naples Lectures, Monographs and Textbooks in Physical Science, Vol. 2 (Bibliopolis, Naples, 1987).

[13] A. Strominger, J. High Energy Phys. 07 (2014) 152; T. He, V. Lysov, P. Mitra, and A. Strominger, J. High Energy Phys. 05 (2015) 151; M. Campiglia and A. Laddha, J. High Energy Phys. 12 (2015) 094; S. W. Hawking, M. J. Perry, and A. Strominger, Phys. Rev. Lett. 116, 231301 (2016); J. High Energy Phys. 05 (2017) 161; A. Strominger, arXiv:1703.05448.

[14] M. Mirbabayi and M. Porrati, Phys. Rev. Lett. 117, 211301 (2016); R. Bousso and M. Porrati, Classical Quantum Gravity 34, 204001 (2017); Phys. Rev. D 96, 086016 (2017).

[15] S. K. Wong, Nuovo Cimento A 65, 689 (1970).

[16] A. P. Balachandran, G. Marmo, and A. Stern, Nucl. Phys. B162, 385 (1980); A. P. Balachandran, G. Marmo, A. Stern, and B. S. Skagerstam, Phys. Lett. 89B, 1991 (1980); A. P. Balachandran, G. Marmo, B.-S. Skagerstam, and A. Stern, Gauge Symmetries and Fibre Bundles, Lecture Notes in Physics 188 (Springer-Verlag, Berlin, 1982).

[17] N. M. J. Woodhouse, Geometric Quantization (Clarendon, Oxford, 1992); J. Sniatycki, Geometric Quantization and Quantum Mechanics (Springer-Verlag, Berlin, 1980); P. Woit, Quantum Theory, Groups and Representations: An Introduction, http://www.math.columbia.edu/\%7Ewoit/QM/qmbook .pdf; V.P. Nair, Elements of Geometric Quantization \& Applications to Fields and Fluids, Lectures at the Second Autumn School on High Energy Physics and Quantum Field Theory, Yerevan, Armenia, 2014, arXiv:1606.06407. 